\title{
Radiation risk from cardiac CT and nuclear cardiology: Addressing concerns with innovative solutions
}

\author{
Andrew J. Einstein, MD, PhD
}

\section{INTRODUCTION}

The problem of radiation exposure associated with nuclear cardiology and cardiac computed tomography has received considerable attention in recent years. This issue of the Journal includes four review articles based on a symposium addressing this topic. Entitled "Radiation Risk from Cardiac CT and Nuclear CardiologyAddressing Concerns with Innovative Solutions," this session was held as part of the 15th Annual Scientific Session of the American Society of Nuclear Cardiology on September 24, 2010.

Fazel and Shaw ${ }^{1}$ begin the series by addressing essential principles of radiological protection: education, justification, and optimization. The principle of justification implies that when activities involving a change in the level of radiation exposure, for example performing a myocardial perfusion imaging exam, are being considered, the decision-making process should explicitly include the change in radiation detriment. The principle of optimization is the process of keeping the likelihood of incurring exposure, the number of people exposed, and the magnitude of individual doses as low as reasonably achievable, i.e. ALARA. ${ }^{2}$ Following is an article by Williams and Ballapuram, ${ }^{3}$ who clarify terminology related to radiation exposure, such as the effective dose, classify types of radiation effects, and provide comparative estimates of radiation doses from various nuclear cardiology procedures. Adhering to the ALARA principle is virtually impossible without a clear understanding and consistent use of such terminology.

The two remaining articles focus on the practical implementation of the principle of optimization to cardiac computed tomography and nuclear cardiology.

From the Columbia University Medical Center, New York, NY.

These review articles are based on presentations made at the American Society of Nuclear Cardiology 2010 meeting, Philadelphia, PA, September 23-26, 2010.

Reprint requests: Andrew J. Einstein, $\mathrm{MD}, \mathrm{PhD}$, Columbia University Medical Center, New York, NY; andrew.einstein@columbia.edu. J Nucl Cardiol 2011;18:561.

$1071-3581 / \$ 34.00$

Copyright $(\subset 2011$ American Society of Nuclear Cardiology. doi:10.1007/s12350-011-9424-6
Small et al. ${ }^{4}$ cover computed tomography, addressing approaches such as reducing the x-ray tube output, minimizing the duration of exposure, limiting the amount of tissue irradiated, and improving the x-ray beam focus. In addition, they cover some emerging methods, such as iterative image reconstruction, highpitch helical scanning, and improved detector elements, which will potentially enhance the magnitude of dose reduction using these general approaches. Henzlova and Duvall $^{5}$ focus on SPECT myocardial perfusion imaging, including optimization strategies such as avoidance of dual-isotope protocols, utilization of stress-only imaging, and use of novel technologies such as advanced image reconstruction algorithms combining iterative reconstruction with resolution recovery and high-efficiency cameras incorporating multiple solid-state cadmium zinc telluride detectors. In discussion, they point out that the myocardial perfusion imaging community has been much less vigorous in dose reduction efforts than has been the cardiac CT community. Let us hope that symposia and publications such as these will lead to better radiation protection for nuclear cardiology patients.

\section{Disclosure}

Dr Einstein has received research grants from GE Healthcare and Spectrum Dynamics.

\section{Reference}

1. Fazel R, Shaw LJ. Radiation exposure from radionuclide myocardial perfusion imaging: Concerns and solutions. J Nucl Cardiol 2011;18. doi:10.1007/s12350-011-9403-y.

2. The 2007 Recommendations of the International Commission on Radiological Protection: ICRP publication 103. Ann ICRP 2007;37:1-332.

3. Williams KA, Ballapuram K. Radiation exposure in diagnostic imaging-use, misuse, or abuse? Part I: The background and science of medical radiation. J Nucl Cardiol 2011;18. doi: 10.1007/s12350-011-9402-z.

4. Small GR, Kazmi M, deKemp RA, Chow BJW. Established and emerging dose reduction methods in cardiac computed tomography. J Nucl Cardiol 2011;18. doi:10.1007/s12350-011-9400-1.

5. Henzlova MJ, Duvall WL. The future of SPECT MPI: Time and dose reduction. J Nucl Cardiol 2011;18. doi:10.1007/s12350011-9401-0. 